\title{
GETTING CLOSER TO THE THINGS WE ARE LOOKING FOR - A PROPOSAL FOR ASPECT-DRIVEN TEXT ANALYSIS
}

\author{
Kurt Englmeier and Matthias Schneider \\ Schmalkalden University of Applied Science, Faculty of Computer Science \\ Blechhammer, 98574 Schmalkalden, Germany
}

\begin{abstract}
Locating facts in texts is still a challenge for retrieval systems. Instead of measuring the relevance of the entire text for a search query, the focus of our approach of fact retrieval and text mining is on spotting only essential parts of text. We propose to enrich search queries by aspects supporting highly precise retrieval. An aspect denotes the unique interpretation of a set of words that appear in close proximity. We conceive an aspect representation as a hierarchical structure composed by its semantic elements that, in turn, constitute the aspect's implicit meaning.

In this paper, we present the definition and application of aspect blueprints that serve as standardized representations of aspects. Our prototypical system Contexter operates on these blueprints and locates facts in texts. In a semi-automatic way, the system tries to detect variants of blueprints that the user can confirm or reject. Here, we illustrate our approach and system in the realm of economic information.
\end{abstract}

\section{KEYWORDS}

Information Retrieval, Text Analysis, Text Mining, Aspects, Named Entities, Bag of Words, Blueprints

\section{INTRODUCTION}

Whatever information retrieval system (IRS) we use to satisfy our information need, we more or less have the same recurring user experience: We enter a couple of keywords, probably select one or two additional search terms recommended by the system, and get an overwhelming list of retrieval results. Many times, we encounter the things we were looking for among the first entries on the list. At least, we assume that the list entries point to the information we require. Quite often, the cumbersome and awkward part of our search starts right here, when we have to sift through all presumably relevant documents in order to find the information we need. This process can get quite time-consuming when our required information is located somewhere in large documents. This performance can be acceptable for IRS when humans complete the retrieval process.

In text mining however, the measurement of relevance on document level is insufficient. Text mining tries to classify the content of a text on a more fine-grained level, i.e. on paragraph or even sentence level. Classifying a text on document level can be justifiable in the case of small texts, when its content covers only one or two contextual aspects. If a text covers a broad variety of aspects, its summarization in a single title or a handful of keywords may be not suitable for most text mining as well as information retrieval purposes.

Instead of summarizing the content of a text (or parts of it) by the most important keywords, we propose the assignment of aspect representations to texts on possibly the most fine-grained level. A hierarchical structure composed of terms describes the respective aspect together with its semantic subcomponents. Information retrieval or text mining processes operate on these aspect representations and enable the location (and extraction) of relevant text passages on a more fine-grained level, that is, on paragraph or sentence level. An interesting application area of our approach can be a Legal Tech system that identifies facts in documents like balance sheets, claims, complaints, or contracts and looks up the pertinent legal rules.

In this article, we illustrate our approach in the realm of fact retrieval in economic reports. The work presented here reflects the current state-of-work of our research group of the Schmalkalden University of 
Applied Science. At first, we outline the theoretical and technical background of our approach. A fundamental set of aspect representations are handcrafted. These aspect blueprints may contain named entities, keywords or N-grams of words. We illustrate the syntax for the definition of blueprints and how our prototypical mining system "Contexter" operates on them in order to analyze and classify texts. We also describe the supervised learning processes for a semi-automatic approach to identify further useful aspect blueprints.

\section{RELATED WORK}

Our approach is designed around the paradigm of fact retrieval emphasizing natural language (Balasubramanian et al., 2007; Fuhr, 1992; Keikha et al., 2014; Kolodner, 1982) and the support of users in constructing more complex search queries (White et al., 2007; Nguyen et al., 2018).

A feature in a text is associated with a token delimited by white space. (Weiss, 2015, p. 27) Even though tokens reference mostly words, they can also address numerical data, codes, or symbols. Each feature denotes, thus, a particular and mainly unique meaning or aspect of a word. In some cases, it can be helpful to consider multiword features. "European Union" or "U.S. Dollar" are examples for multiword features. The corresponding tokens represent a unique meaning, but only when occurring together. Names of persons are also examples of multiword features. In contrast to the previous examples, the sequence of tokens addressing names need not necessarily be consecutive. There can even be a middle name that is referenced in one feature and omitted in the other, but both multiword features address the same person. The classification of text using multiword features has a long tradition (Acosta et al., 2011; Calzolari et al., 2002; Papka and Allan, 1998). From a different point of view, multiword features can also be considered as word n-grams or n-words (Huston et al., 2012).

An instrument that proved to be useful in our context are Bag of Words (BoW). They contain keywords indicating institutions like Wall Street, Dow Jones, or White House. We can imagine a variety of theme-specific BoWs (for locations, names, sentiments etc.) applicable in a given context (Woods, 1996).

The concept of feature, however, is not restricted to words. Named entities are probably the most essential ingredients for the composition of features. At first, in combination with words they identify common patterns in texts reflecting time, prices, distances, and the like. A currency, for example, is usually represented by a symbol or code. The temperature is usually indicated by a numerical expression with an adjacent symbol for degrees in Kelvin, Celsius, or Fahrenheit. Named entities also help to standardize factual information and to abstract away the different forms of expressions for essentially the same thing. Named entities indicate that is probably more suitable to talk about multi-expression features that refer to a series of text elements and indicate their meaning.

Named entity recognition (NER) may even take a special role in the identification of elements of BoWs, when special indicative terms appear in close proximity to words with particular characteristics, mainly proper names. Words such as "Mr." or "Health Senator" hint to names of persons. There are further indicative terms pointing to names. For example, the term "by" following the title of an article often leads the list of names authoring that article. The identification of proper names benefits from the analysis of sequential dependencies when bags of words can be produced automatically instead of manually. There are promising approaches to automatically identify names (and other important key expressions) in texts using conditional random fields (CFR) (Sha and Pereira, 2003) or hidden Markov Models (HMMs) (Freitag and McCallum, 2000). A conditional pattern, for example, can be a series of capital letters in parentheses following words starting with capital letters. This pattern may indicate organization names with their respective abbreviations, such as National Institute of Health (NIH) or Korean Electric Power Corporation (KEPCO).

The next important step in the analysis of text is the annotation of features according to the meaning they express (Cowie and Lehnert, 1996; Salton et al., 1997). Annotation has two roles: first, it adds a meaningful term to features, in particular when they contain numeric data. Such patterns, for example, represent dates, percentages, numerical data, distances, and the like. Second, the annotations themselves represent a feature that again can be part of multi-expression feature. When iteratively performed, annotation produces an increasingly more abstract representation of the content it addresses. The target application area of our approach, however, is the location of text passages that are strongly related. Legal information retrieval systems (Heo et al, 2017, for instance, try to find pertinent rules regulations for facts or issues mentioned in a complaint, claim, contract, etc. 


\section{ASPECTS AND THEIR BLUEPRINTS}

\subsection{The Representation Structure of Aspects}

Features stand for a precise meaning represented by their constituting expressions. Aspects, on the other hand, denote the interpretation of text features (Singh and Croft, 2016). The expression "500 $€$ " means a precise amount of money and "2.4 GW" a precise amount of energy. An aspect representation tries to interpret the content of its feature as precisely as possible. The amount of money, for instance, can be a price tag or a characteristic of a money transfer. The more surrounding information we take into account the more complex, but also the more correct gets the definition of an aspect and the interpretation of its constituting features. If a feature like "costs" is adjacent to the expression of the amount of money, then the features together constitute the aspect "price tag". The price tag, in turn, may stand for a bargain or its contrary. This again may be part of a purchase, which usually includes vendor, buyer and further characteristics.

With the growing integration of aspects, we achieve a hierarchy with the most abstract description on its top. Let us consider the aspect "investment in nuclear energy production". This aspect consists of the two parts "investment" and "nuclear energy production". The aspect "investment", in turn, may be composed by constituting elements such as "investor", "investment object", and "price". All of these aspects can be further detailed, and so on. For each aspect, we obtain more or less complex, hierarchical representations. Aspects on the fine-grained level of the hierarchy may cover a few adjacent features. With the growing level of abstraction, an aspect may span an entire phrase, paragraph, page, or even more. The aspect of the purchase of a real estate can easily span an entire document of a dozen of pages.

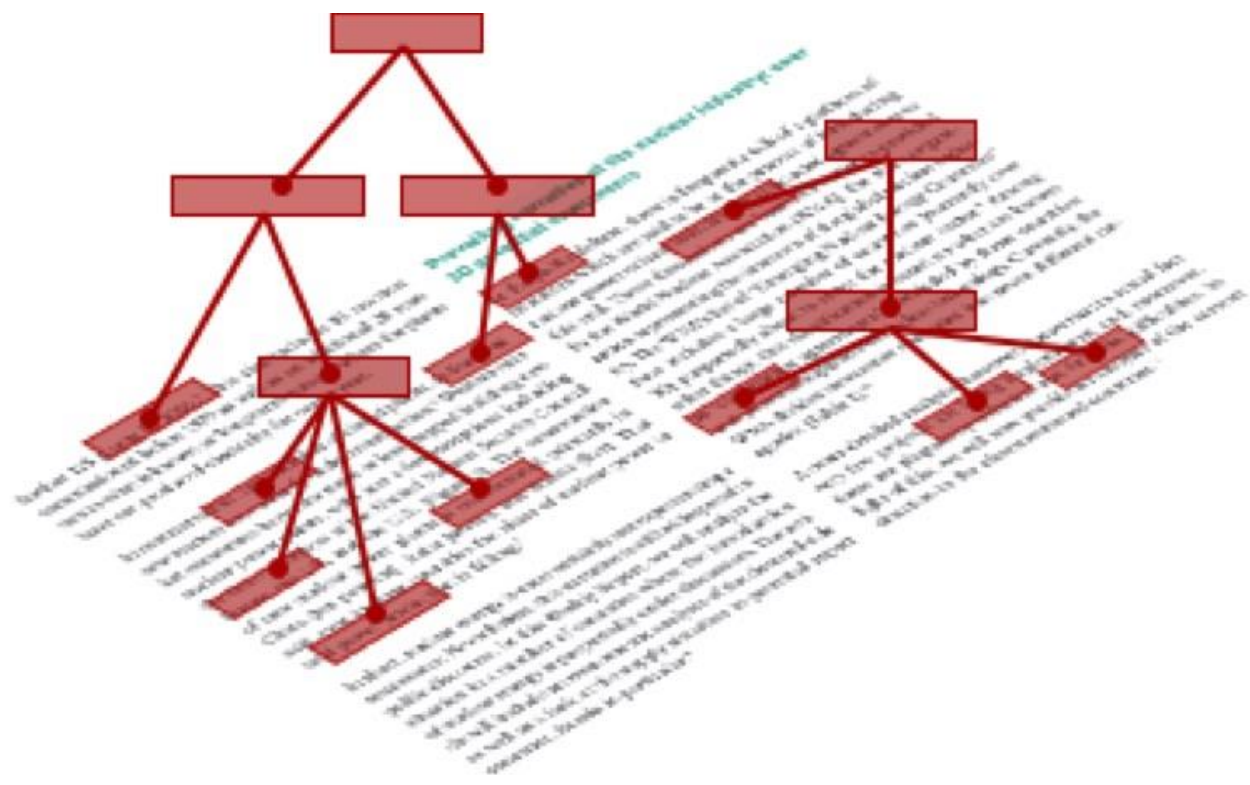

Figure 1. Schematic presentation of an aspect hierarchy and its subcomponents

The top-level aspect in a hierarchy of aspects represents a fact in an abstract way. It is obvious, that there may be many variations in the representation of this aspect. Variations even start on a fine-grained level. A named entity composed of a numerical expression with an adjoint currency symbol constitutes the aspect "price". There may exist as many variants of this aspect as exist currencies in the world. And even each currency can be denoted in multiple ways (e.g. "U.S. dollar", "USD”, “\$”). 


\subsection{The Syntax of Aspect Blueprints}

The aspect blueprint is the central element in our approach for a combination of text and fact retrieval and for the classification processes in text mining. In order to cover all variants of an aspect, we have to find a suitable blueprint that covers all its possible instances. As reflected in figure 1, the blueprint is basically a structure of expressions where each element may hold one or more

- $\quad$ keywords (in their stemmed forms, including their corresponding synonyms),

- references to BoWs,

- n-grams (as a series of adjacent features),

- $\quad$ named entities, or

- references to further aspects on a more fine-grained level.

Regular expressions are a powerful instrument to detect patterns in all kinds of unstructured data, not only in texts. However, they require special skills and are not easy to handle, in particular, when they are addressing complex, i.e. real-world, patterns. Nevertheless, regular expressions are key to flexible and powerful text analysis.

Our approach adopts regular expressions but shields the users from their complexity by hiding them in a set of labeled expressions starting with keywords, n-grams, and named entities representing things like name, social security number, distance, birthday etc. Instead of defining regular expressions, the users define their blueprints by resorting to these more basic expressions. The users then gradually define more complex expressions by integrating already existing blueprints. The syntax they use in order to define their blueprints is quite simple. It enables the user to describe adjacent elements of an aspect. A blueprint of an order, for example, lists the persons or organizations that act as sellers and buyers, the product, the date, and the price.

Table 1. Operators of the syntax to define aspect blueprint

\begin{tabular}{|c|c|}
\hline Operator & Function \\
\hline • & $\begin{array}{l}\text { The dot indicates strong sequence ("followed by"). The expression } \\
\text { indicated before the dot must be located before the one indicated after the } \\
\text { dot. }\end{array}$ \\
\hline , & $\begin{array}{l}\text { Comma means weak sequence. Some indicated expressions (at least one) } \\
\text { shall appear sequentially in the data. However, they may appear in any } \\
\text { order (inclusive combination). }\end{array}$ \\
\hline ; & $\begin{array}{l}\text { The semicolon is used to indicate an exclusive combination. Just one of } \\
\text { the expressions ought to be located. }\end{array}$ \\
\hline : & $\begin{array}{l}\text { Labeling (annotation) operator: the name after the colon is assigned to } \\
\text { expressions or a group of expressions. }\end{array}$ \\
\hline$(\ldots)$ & $\begin{array}{l}\text { Parentheses serve to indicate a group of expressions. Grouping only } \\
\text { makes sense together with the labeling operator. }\end{array}$ \\
\hline$?$ & $\begin{array}{l}\text { The question mark indicates that an expression or group of expressions is } \\
\text { optional. }\end{array}$ \\
\hline & Keywords are indicated between quotation marks \\
\hline
\end{tabular}

The users may indicate the aspect of "decrease" by the following blueprint: decrease="decline" . fraction,time point.fraction.time point. "fraction" stands for a named entity representing percentages and similar expressions. All representations of a single point in time are represented by the named entity "time point". The keyword "declined" is a placeholder for all synonyms reflecting a decline. Before the Contexter system applies this blueprint to any text, the keywords are implicitly replaced by the stemmed versions of all their synonyms. Stemming is, of course, also applied to the target text beforehand. After the application of this blueprint a piece of text concerning a decrease is marked like this: "The share of nuclear energy in power generation has <decrease>declined from its peak of <fraction>17 percent</fraction> <time point>in <year>1996</year ></time point> to around <fraction>ten percent</fraction $>\quad<$ time point $>$ today $</$ time point $></$ decrease $>$ ". 
A particular aspect can be expressed by different words, much like the type of numerical information (date, price, or growth rate, for instance) can be expressed in different syntactic forms. It is important that a unique title represents each aspect. Each blueprint, in turn, represents not only the semantic architecture of its meaning, but also the different syntactic facets its instances may take in texts.

Whenever an expression of a blueprint refers a specific keyword, all of its applicable synonyms need to be taken into consideration. However, not all possible synonyms are also applicable in every context. In an expression describing a certain amount of money like "to the tune of 12.65 billion U.S. dollars", none of the synonyms of the term "tune" is applicable in this context. In some occasions, it is thus recommendable to consider the applicability of synonyms by considering $\mathrm{n}$-grams. Thorough $\mathrm{n}$-gram analysis reveals, that "to the tune of" have synonyms like "to the amount of" or "add up to".

Figure 2 shows the representation of a piece of text referencing a broader aspect, namely an investment. At first, all instances that meet the qualities of an expression representing a price are identified and marked by the blueprint price=?"price". money . currency. These instances are expected to be composed of an instance matching the blueprint (amount of) "money", a further one addressing the currency and an occasional (leading or trailing) word "price" (or a synonym expression such as "at a cost of"). The expression "price" is optional and thus leaded by a question mark. Keywords are stated in quotes. Internally they are mapped to their standardized (stemmed) forms. Terms without quotes thus refer to the blueprint elements or to BoWs. The dots stand for "close proximity" which can range from "immediately adjacent" to "next blueprint coverage".

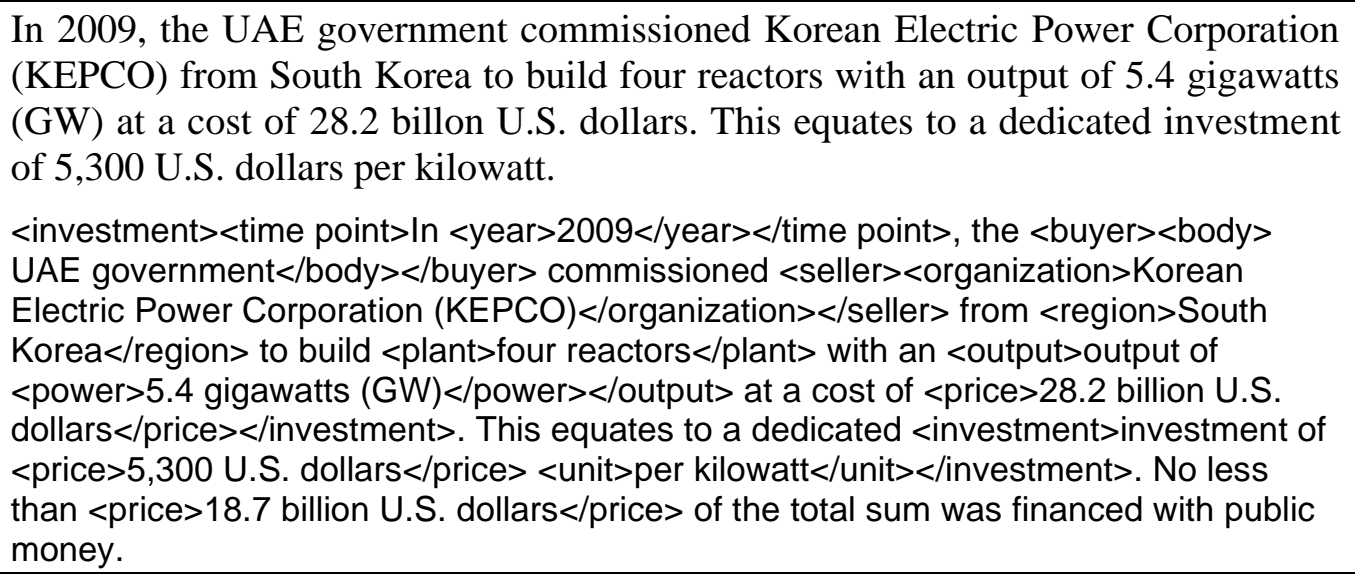

Figure 2. Section of a text and its representation after basic text patterns have been identified and accordingly annotated

\section{THE SEMI-AUTOMATIC PROCESS OF TEXT ANALYSIS}

In the first place, hand-crafted blueprints may appear outdated given the availability of so many powerful automatic information extraction systems that use machine-learning or similar techniques (Etzioni et al., 2008). We believe that the performance of the analysis process benefits from user intervention. The users are good in formulating hypotheses about the things they expect to encounter in texts. This can be the starting point for machines to step in and support the users. The support can take two fundamental forms: First, providing users with a language for the definition of aspect blueprints that is fast to learn and easy to handle and, second, a machine that takes the users' blueprints and automatically discovers further blueprints on its own.

The semi-automatic process takes the blueprints defined by the users, in fact, as seeds and tries to locate similar but new blueprints. The findings are presented to the users that confirm or reject the results of the automatic process. The first group of blueprint elements that benefit from automatic discovery are proper names, usually stored in the BoWs. Proper names pose, in general, a certain challenge to automatic text analysis. By applying identification rules inclined to CFR methods, we can automatically identify proper names to a certain extend. For instance, words starting with uppercase letters and immediately following special terms like "Premier ministre", "Mr.", or "the author" usually indicate that these words are proper 
names of persons. The system can identify names of organizations by applying suitable rules like the ones described here. Some BoWs (for countries, for instance) can also be imported from external sources. However, there always remain proper names in text that are not covered by external sources. These names need to be detected and added to the BoWs.

The more interesting learning process for our system targets the discovery of blueprints. Each set of blueprint elements is annotated by a title reflecting the overarching aspect of the elements. By repeatedly applying this process the blueprint gets more layers and covers a growing text area. The blueprint then resembles a hierarchy with a general representation of covered text on its top and growing specialized representations towards its bottom. In principle, the system takes the user-defined blueprints as seeds and tries to find further applicable blueprints that are similar to the seeds. This title summarizes the content of all blueprint components on its underlying layer. It thus abstracts away the content details of the sub-component layer it stands for. Each such blueprint can be a constituting element in the next layer of abstraction. If blueprints can be applied to text, they cover a certain text area. It can be a sentence, a paragraph, or a larger text section.

The basic idea of the semi-automatic process is that the system modifies existing blueprints on its own and test them against the text base. If the system finds text instances that match the modified blueprint, it reports this blueprint back to the users that confirm or reject it. When the users confirm a newly found blueprint is added to the collection of existing aspect blueprints. The system modifies the blueprint

- by trying to substitute its subcomponents one by one or

- $\quad$ by adding new subcomponents that the fringe of the blueprint (figure 3).

The process of substitution starts with blanking out a subcomponent. The remaining blueprint is then applied to the text base. Whenever it matches, the text passage between the subcomponent adjacent to the blanked-out subcomponent gets further investigated. The system tests if there is an already existing blueprint in the collection that can be applied to this piece of text and, thus, serves as new subcomponent.

Similarly, the system tests if it can add a blueprint at the fringes of the blueprint's text coverage. The coverage area can be a fraction of a phrase, an entire phrase, more than one phrase, or even one or more paragraphs. The system ventures out into the adjacent text, tries to find an applicable blueprint and considers it as new subcomponent. Depending on the size of the coverage area, the system explores the surrounding sentence, couple of sentences, or paragraph. The text, the system explores, may not be covered by another blueprint that has at least of one level of subcomponents. Texts covered by hierarchical compositions of blueprints are further limits for the system's exploration into yet uncovered text areas. Single aspect blueprints that are not building blocks of other blueprints may be added.

Eventually, the system presents all new blueprints to the users that, in turn, select the ones that make sense and reject the others. The probably more challenging task for the users is the selection of suitable, consistent, and unambiguous titles for the blueprints. Moreover, the title once defined by the individual user needs to be institutionalized. The titles of the blueprints reflect the semantic picture of all aspects an organization is dealing with in its information management. There are standardized concept hierarchies available like schema.org or the nomenclatures of statistical offices. However, they cover only small fractions of the information ecosystem addressed by an organization.

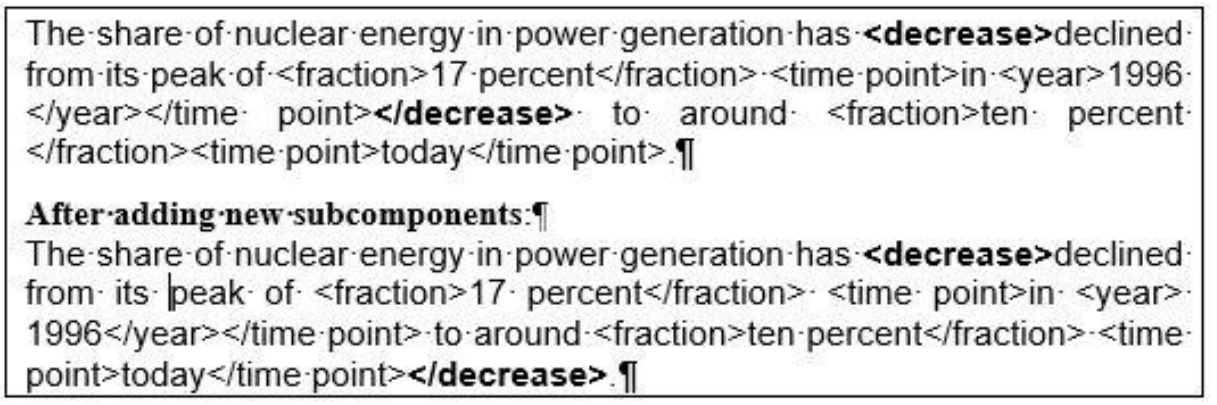

Figure 3. Example of the expansion of an already existing blueprint by examining the text area beyond the fringes of the actual text coverage 
A further objective of our approach is a stronger involvement of humans in the development and management of text mining tools and, in general, to enhance the adoption of this technology on a broader scale. This involvement results in a more active role of the users in designing, controlling, and adapting the learning process that feeds, in this case here, the automatic detection of facts in text. The syntax for the definition of a blueprint is easy to learn. Even users without technical background are in the position to write definitions for aspect blueprints. In the next phase of the development of our prototype, the users will be involved more closely in the training of the semi-automatic processes to detect blueprints that come semantically close to existing definitions.

\section{REFERENCES}

Acosta, O.C., Villavicencio, A., and Moreira, V.P. (2011) 'Identification and Treatment of Multiword Expressions Applied to Information Retrieval', Proceedings of the Workshop on Multiword Expressions: From Parsing and Generation to the Real World, pp. 101-109.

Balasubramanian, N., Allan, J. and Croft, W.B. (2007) 'A comparison of sentence retrieval techniques', Proceedings of the 30th annual international ACM SIGIR conference on Research and development in information retrieval, pp. 813-814.

Calzolari, N., Fillmore, C.J., Grishman, R., Ide, N., Lenci, R., Macleod, C., and Zampolli, A. 'Towards Best Practice for Multiword Expressions in Computational Lexicons', Proceedings of the Third International Conference on Language Resources and Evaluation (LREC 2002), pp. 1934-1940.

Cowie, J. and Lehnert, W. (1996) "Information Extraction". Communications of the ACM 39(1), pp. 80-91.

Etzioni, O., Banko, M., Soderland, S., and Weld, D.S. (2008) "Open information extraction from the web", Communications of the ACM 51(12), pp. 68-74.

Freitag, D. and McCallum, A. (2000) "Information Extraction with HMM Structures Learned by Stochastic Optimization", Proceedings of the Seventeenth National Conference on Artificial Intelligence and Twelfth Conference on Innovative Applications of Artificial Intelligence, pp. 584-589.

Fuhr, N. (1992) 'Integration of probabilistic fact and text retrieval', Proceedings of the 15th annual international ACM SIGIR conference on Research and development in information retrieval, pp. 211-222.

Heo, S., Hong, K., and Rhim, Y.-Y. (2017) "Legal Content Fusion for Legal Information Retrieval", Proceedings of the 16th Edition of the International Conference on Articial Intelligence and Law, pp. 277-281.

Huston, S., Culpepper, J.S., and Croft, W.B. (2012) 'Sketch-Based Indexing of n-Words', Proceedings of the 21st ACM International Conference on Information and Knowledge Management, pp. 1864-1868.

Keikha, M., Park, J.H., Croft, W.B. and Sanderson, M. (2014) 'Retrieving Passages and Finding Answers', Proceedings of the 2014 Australasian Document Computing Symposium, pp. 81-84.

Kolodner, J.L. (1982) 'Requirements for natural language fact retrieval', Proceedings of the ACM '82 conference, pp. 192-198.

Nguyen, A.T., Kharosekar, A., Krishnan, S., and Krishnan, S. (2018) 'Believe it or not: Designing a Human-AI Partnership for Mixed-Initiative Fact-Checking', Proceedings of the 31st Annual ACM Symposium on User Interface Software and Technology, pp. 189-199.

Papka, R. and Allan, J. (1998) 'Document classification using multiword features', Proceedings of the seventh international conference on Information and knowledge management, pp. 124-131.

Salton, G., Allan, J., Buckely, C., and Singhal, A. (1997) "Automatic Analysis, Theme Generation, and Summarization of Machine-Readable Texts", in: Karen Sparck Jones and Peter Willett, Readings in Information Retrieval, San Francisco: Morgan Kaufmann, pp. 478-483.

Sha, F. and Pereira, F., (2003) "Shallow Parsing with Conditional Random Fields", Proceedings of the HLT-NAACL conference, 2003, pp. 134-141.

Singh, M. and Croft, W.B. (2016) „Iterative Search using Query Aspect“, Proceedings of the 25th ACM International on Conference on Information and Knowledge Management, pp. 2037-2044

Weiss, S.M., Indurkhya, N., Zhang, T. (2015) Fundamentals of Predictive Text Mining, $2^{\text {nd }}$ edn. London: Springer.

White, R.W., Drucker, S.M., Marchionini, G., Hearst, M., and Schraefel, M.C. (2007) 'Exploratory search and HCI: designing and evaluating interfaces to support exploratory search interaction', CHI '07 Extended Abstracts on Human Factors in Computing Systems, 2007, pp. 2877-2880.

Woods, W.A., (1996) “Context-Sensitive Parsing”. Communications of the ACM 13(7), pp. 413-445. 